\title{
Tidal airway closure in asthma and COPD
}

\author{
Claudio Tantucci, Laura Pini \\ Respiratory Medicine, Department of Clinical and Experimental Sciences, University of Brescia, Italy
}

\begin{abstract}
Functional closure of small airways can occur during tidal breathing above functional residual capacity (FRC) both in asthma and chronic obstructive pulmonary disease (COPD) patients, especially during exacerbations. Such event has several noxious consequences on gas exchange, airway hyperresponsiveness and mechanical stress and strain within lung tissue and airway wall, mostly due to increase in ventilation heterogeneity. The availability of simple functional tests based on sequential measurements of lung volumes (i.e., FRC), by plethysmography and dilutional techniques may reveal and monitor easily tidal airway closure that can be and should be treated with the aim of abolishing or at least reducing this dangerous condition.
\end{abstract}

\section{Introduction}

The lumen of bronchioli that lack a cartilaginous support despite the presence of a relatively increased amount of smooth muscle in their wall, is preserved through the small airway-lung parenchyma interdependence and the local production of tension-active substance $[1,2]$.

The interdependence is function of both lung elastance and integrity of alveolar attachments on the adventitia of the small airways [1]. The elastic forces related to surface tension developed at the air-epithelial lining fluid interface inside the small airways lumen that act to close it, are reduced by the surfactant produced by the Clara cells [2].

Normally, these factors are able to maintain the small airways open during tidal breathing with the exception in the older subjects where, because of age-related loss of the lung elastic recoil pressure, the closing capacity may occur above the end expiratory lung volume (i.e.,

Corresponding author: Dr. Laura Pini, Respiratory Unit, Department of Clinical and Experimental Sciences, University of Brescia, Piazzale Spedali Civili 1, Brescia, Italy. E-mail: laura.pini@unibs.it

Key words: Tidal airway closure; FRC; plethysmography; dilutional techniques.

Received for publication: 4 November 2016

Accepted for publication: 14 November 2016

(C) Copyright C. Tantucci and L. Pini, 2016

Tipografia PI-ME Editrice, Italy

Monaldi Archives for Chest Disease Pulmonary Series 2016; 83:788

doi: 10.4081/monaldi.2016.788

This article is distributed under the terms of the Creative Commons Attribution Noncommercial License (by-nc 4.0) which permits any noncommercial use, distribution, and reproduction in any medium, provided the original author(s) and source are credited. the plethysmographic functional residual capacity), earlier in lying position and then also in sitting position [3]. In case, the dependent small airways tend to stay closed during tidal breathing because their negative transmural pressure is not counterbalanced enough by the decreased lung elastance. This phenomenon is believed to generate low ventilation/perfusion ratios and/or intrapulmonary shunt in the dependent lung regions, causing age-related progressive hypoxemia with increased alveolar-arterial pressure difference [4].

\section{Tidal airway closure}

The patency of small airways during tidal breathing is crucial to preserve homogeneous distribution of ventilation that narrows the regional V/Q ratios, improves gas exchange, reduces airway hyper-responsiveness and limits stress forces and strain within the lung tissues. Conversely, their functional closure above functional residual capacity (FRC) is going to produce the opposite, leading to a vicious circle that may characterize the severity of obstructive lung diseases such asthma or chronic obstructive pulmonary disease (COPD) (Figure 1).

In particular, the mechanical load following the patchy closure of small airways during tidal breathing has been neglected for long time, but recently this has been clearly appreciated that it can sustain lung parenchyma injury directly or by inducing further and persistent inflammation [5]. Accordingly, shear forces and distortion, due to heterogeneous distribution of tidal volume, that tend to prevail within those lung regions served by small airways still open, will induce a relevant mechanical stress with its biological effects [6,7]. On the other hand, every spontaneous sight or deep inhalation by increasing the volume-related elastic recoil pressure of the lung can reopen transiently the small airways previously closed ("pop-up" phenomenon) with possible mechanical stress/injury on their epithelial layer $[8,9]$. Subsequent further inflammation and remodeling in their wall will ensue sustained by different pathways not entirely well defined yet, involving NO production, cytokines and chemokines release and growth factors synthesis $[9,10]$.

Therefore, the consequences of uneven closure of small airways during tidal breathing possibly due to intraluminal mucus exudates accumulation, submucosal and adventitial inflammation/edema of the airway wall, bronchoconstriction, bronchiolar remodeling and poor small airway-parenchyma interdependence may be very dangerous on a long term and need to be avoided keeping the small airways open as much as possible during the day and the night.

All these features can be observed in asthma and in COPD patients, especially during exacerbations and in fact, small airways closure during tidal breathing is expected to happen in these circumstances. Many among the above mentioned factors leading to this condition are irreversible, but some can be controlled by the available treatments. Thus, we need to recognize such abnormal functional aspect that is pathologically and clinically relevant for the patients, either when occurs naturally or elicited by pharmacological testing. 


\section{Tidal airway closure : adverse effects in asthma and COPD}

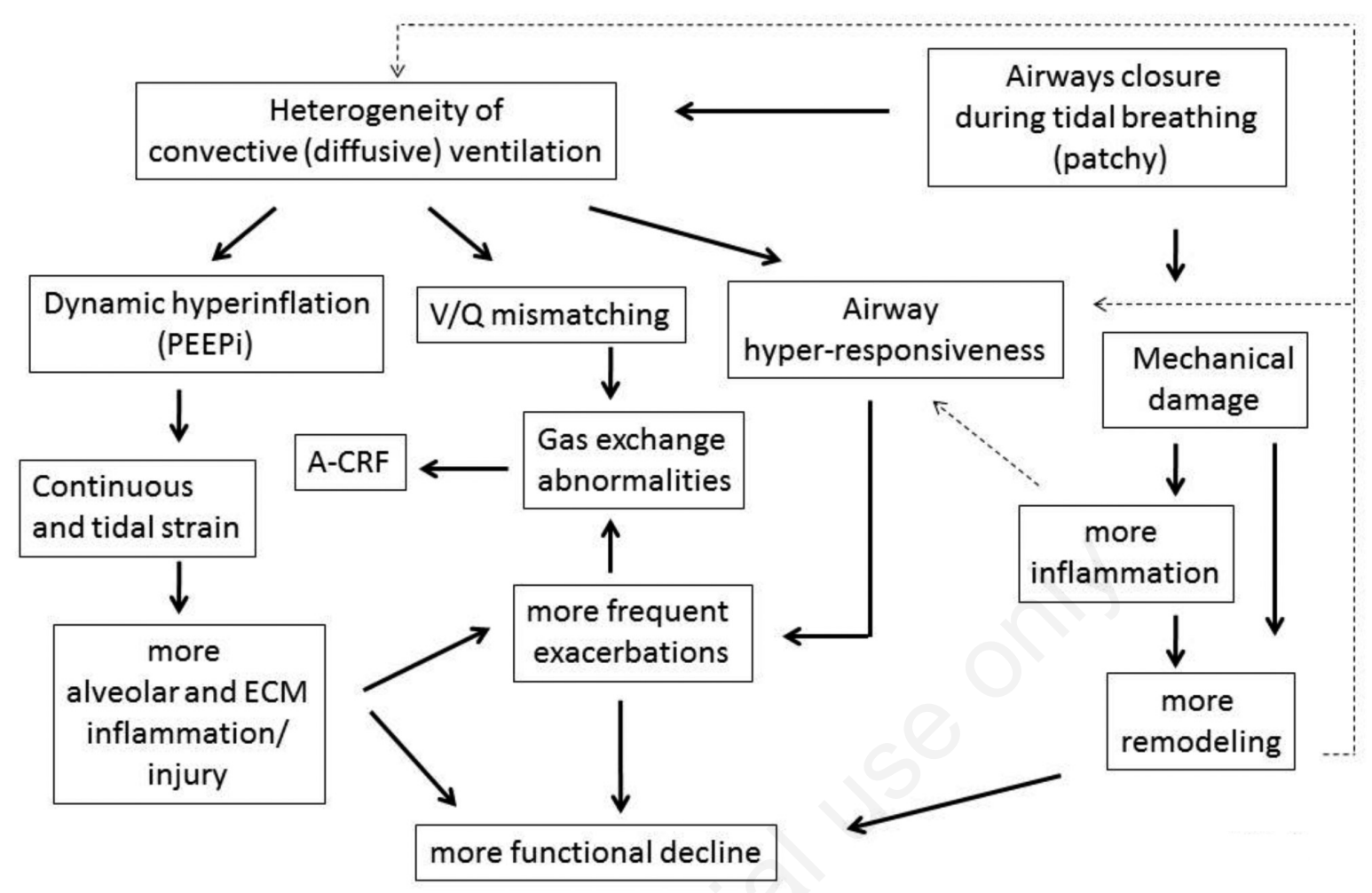

Figure 1. Schematization of the mechanisms underlying the adverse consequences of airway functional closure during tidal breathing in asthma and COPD.

\section{Pulmonary function measurements}

In asthma exacerbations, during sudden attacks or progressive worsening of the clinical condition, the strong and/or sustained contraction of airway smooth muscle, together with mucus plugging, in the presence of uncontrolled, underlying adventitial inflammation may induce closure of airway lumen during tidal breathing, especially in the distal airways [11]. In fact, under these circumstances, the intrinsic load provided by the interdependence mechanism may be lost or greatly reduced due to the airway-lung parenchyma uncoupling caused by periadventitial inflammatory fluid accumulation [12] and remodeling process involving peculiar rearrangement of proteoglycan deposition within extra-cellular matrix of the airway wall $[13,14]$.

This has been demonstrated for both large (internal diameter $>2 \mathrm{~mm}$ ) and small (internal diameter $<2 \mathrm{~mm}$ ) airways during induced bronchoconstriction or natural events throughout dynamic imaging of the lungs obtained by using ultrafine radioactive aerosol and SPECT technique [15] or hyperpolarized helium-3 magnetic resonance [16].

Pulmonary function measurements have been also suggested as markers of peripheral airway closure in asthma such as the reduction of FVC with little or no change of $\mathrm{FEV}_{1} / \mathrm{FVC}$ ratio either during natural or induced airway obstruction or the absence of a "plateau" in the $\mathrm{FEV}_{1}$ decrease during progressive methacholine or histamine provoked broncho-constriction [17]. However, these parameters that are associated with a condition of severe asthma, do not detect airway closure during tidal breathing. Recently, the determination of an appre- ciable difference between FRC sequentially measured by body plethysmography and multibreath helium dilution technique has been proposed as simple and widely available method to indirectly assess the occurrence of tidal airway closure. The reasoning is that body plethysmography measures all compressible air in the lung at FRC, while the dilution technique using multibreath helium in the closed circuit measures the lung volume that can be ventilated at FRC. Normally these measures are identical, but may differ in the presence of an obstructive ventilator defect when helium cannot mark some lung regions that are not directly ventilated because served by functionally closed airways [18].

This method was adopted during methacholine-induced bronchoconstriction in asthmatic patients to show that for a given fall of $\mathrm{FEV}_{1}$, despite similar degree of dynamic pulmonary hyperinflation, some of them suffered from tidal airway closure, as judged by the substantial difference between FRC,Pleth-FRC,He, reflecting a more severe peripheral airway inflammation and remodeling (Figure 2) [19].

Such information seems relevant because these patients could not be distinguished from the other ones who exhibited no difference between FRC,Pleth-FRC,He, according to baseline lung function and level of airway hypersensitivity, as measured by $\mathrm{PD}_{20} \mathrm{FEV}_{1}$, or degree of air trapping (increase in plethysmographic residual volume) and reduction of FVC at comparable maximum level of broncho-constriction [19].

This method can be easily applied in clinical practice to follow the response to the medical treatment offered to exacerbated asthmatic patients, looking at closure and subsequent reopening of small airways during tidal breathing, as shown by the example illustrated in Figure 3. 

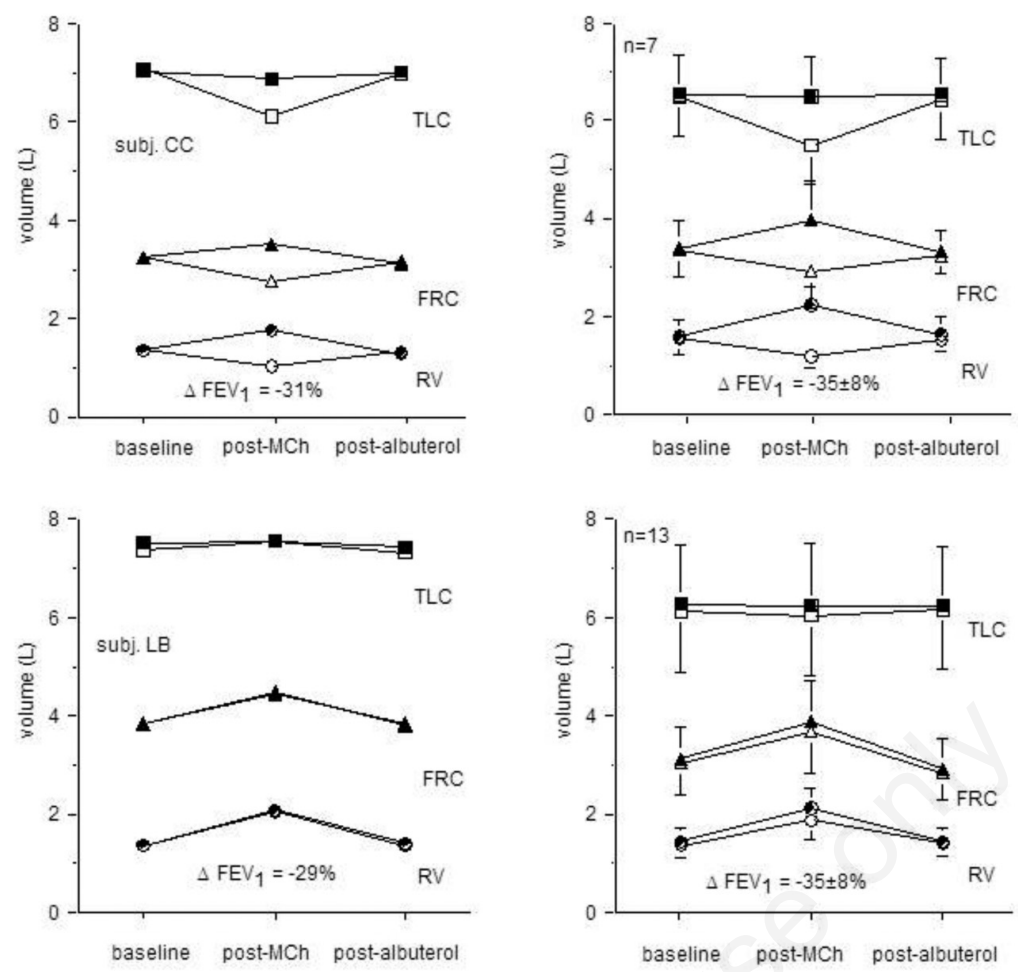

Figure 2. FRC (triangles), RV (circles) and TLC (squares) sequentially measured by plethysmography (black symbols) and multibreath helium dilutional technique (white symbols) at baseline, at the maximum of MCh-induced bronchoconstriction and after recovery following albuterol are shown in two representative asthmatics (left panels) and in two groups of asthmatic subjects (right panel), divided according to decrease (top panels: Group II) or increase (bottom panels: Group I) of FRC,He at the end of MCh challenge. The Mch-induced bronchoconstriction is associated with marked Diff.FRC,Peth-FRC,He in Group II, suggesting substantial tidal airway closure only in these asthmatics.

\section{Bronchial asthma}

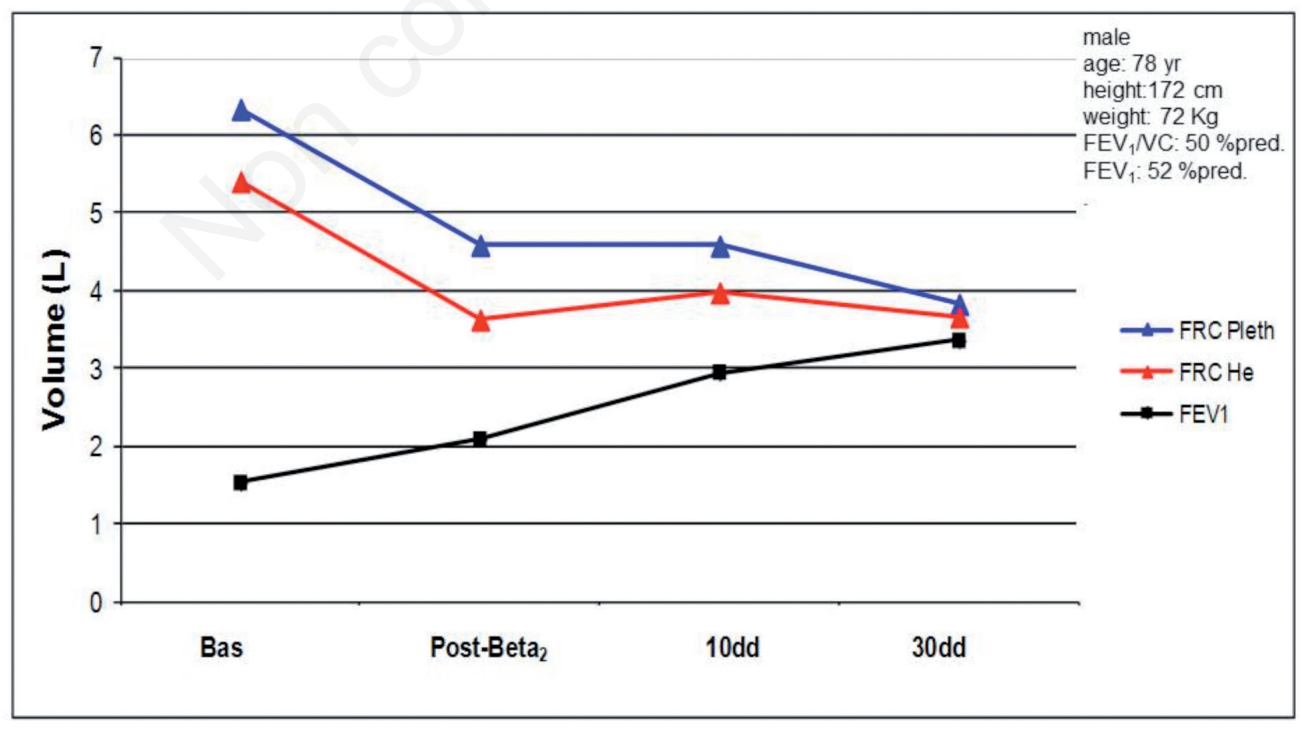

Figure 3. Changes in FRC,Pleth and FRC,He observed in a representative asthmatic patients with an acute exacerbation, at baseline and during therapeutic follow-up. The substantial difference between FRC,Pleth and FRC,He at the beginning, reflecting the presence of tidal airway closure, is completely abolished with treatment, suggesting a complete reopening of small airways during tidal breathing. Note the decrease in dynamic pulmonary hyperinflation with treatment as demonstrated by the plethysmographic FRC reduction. Bas = baseline; dd

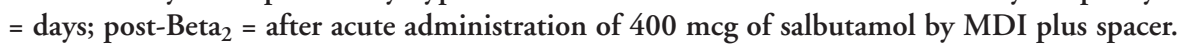




\section{Plethysmographic functional residual capacity}

In COPD patients substantial discrepancy between FRC,Pleth and FRC,He can be observed more easily, even during stable conditions, especially when the airflow obstruction is severe-to very severe. In part this can reflect patchy closure, in part dependent closure of small airways during tidal breathing. The latter originates from a high closing capacity that may exceed plethysmographic FRC, when the closing volume (CV) is greater than expiratory reserve volume (ERV), because of decreased lung elastance due to older age of COPD patients and the emphysematous component of the disorder. In any case, a huge ventilatory heterogeneity can be envisaged under these conditions that associated to dynamic pulmonary hyperinflation often found in these patients also at rest can sustain and increase both lung and systemic inflammation [20,21].

The reversible factors of tidal airway closure such as increased cholinergic tone and bronchoconstriction, submucosal edema and eosinophilic inflammation, intraluminal mucus exudates mostly observed in COPD patients suffering from prevalent chronic bronchiolitis must be adequately treated, trying to keep the small airways open during normal breathing as much as possible throughout all the day.

In a small group of stable COPD patients with moderate-to-severe airflow obstruction showing a baseline marked difference between FRC,Pleth and FRC,He, the regular administration for a 8 week-period of an ultra-LABA bronchodilating agent such as indacaterol by DPI, was able not only to deflate these patients by significantly reducing plethysmographic FRC, but also to almost completely reverse tidal airway closure, as suggested by the similar measurements of FRC with both techniques obtained after treatment (unpublished data) (Figure 4).
This is not the usual result following bronchodilators in more advanced COPD patients where the difference between FRC,Pleth and FRC,He can be definitely reduced but never abolished (Figure 5). Nevertheless, diminishing any source of ventilatory heterogeneity may reduce stress forces and strain within lung structures, limiting the mechanical damage and the consequent inflammation/remodeling of lung tissue. Moreover, the pharmacological stent of small airways, maintaining them open where this is attainable, can impede the repetitive volume-related "opening and closing" process during tidal breathing and the likely associated mechanical epithelial injury. Therefore, the following inflammation and/or remodeling of the small airways wall might be less extensive with improvement of gas exchange and airway hyperresponsiveness and with long-term positive effects on lung function decline and risk of COPD exacerbations.

\section{Conclusions}

In summary, both in asthma and COPD patients small airway closure can occur during tidal breathing and may be easily demonstrated with simple pulmonary function testing implying lung volume measurements, rather than expensive and difficult techniques based on dynamic imaging of ventilation heterogeneity. The unfavorable pathophysiological and clinical implications of this condition begin to be understood, but more evidence is necessary. Nonetheless, the abolition or reduction of tidal airway closure seems very useful and should be firmly achieved and persistently maintained.

\section{After treatment with bronchodilator (Indacaterol $150 \gamma$ )}

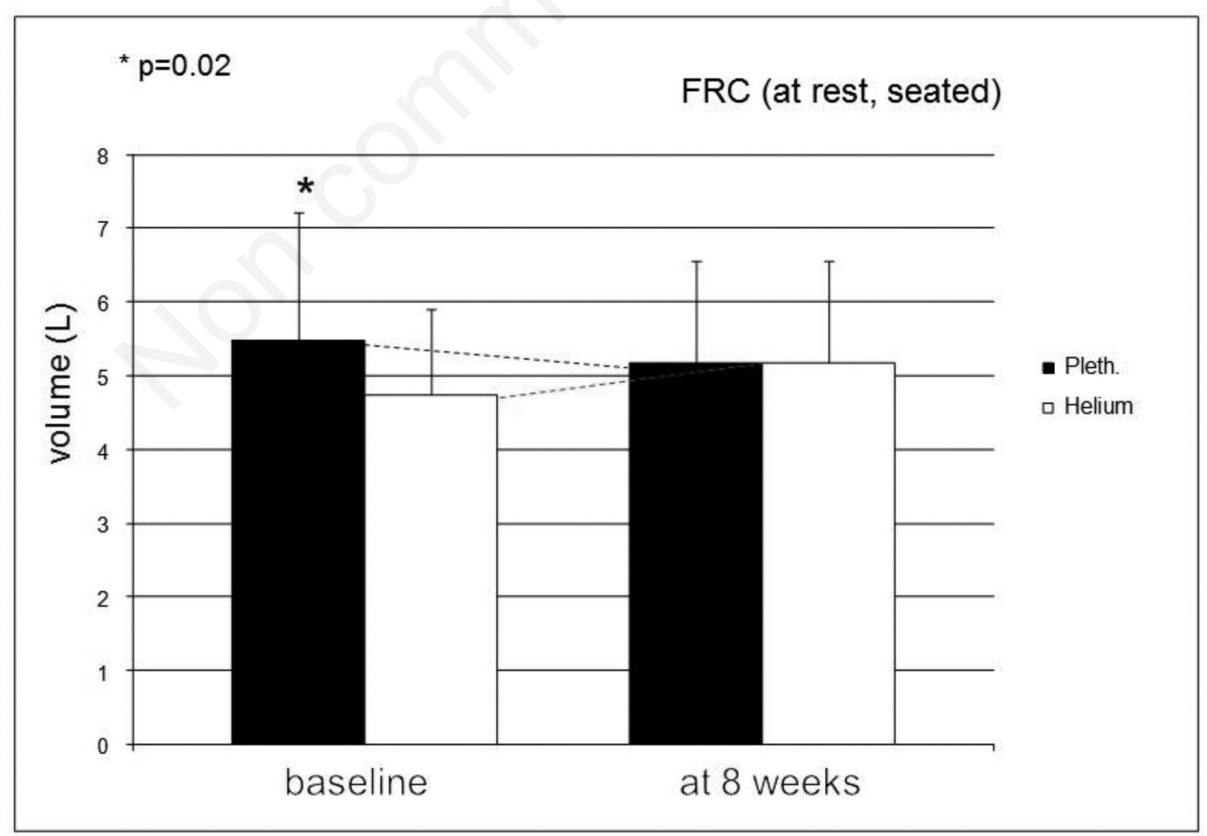

personal data

Figure 4. FRC,Pleth and FRC, He measurements at baseline and after 8-week period of regular therapy with ultra-long acting beta 2 -agonist (Indacaterol, $150 \mathrm{mcg}$ by DPI) in a group of 8 stable COPD patients with moderate airflow obstruction $\left(\mathrm{FEV}_{1}=53 \pm 12 \%\right.$ pred.). The difference between FRC,Pleth and FRC,He at baseline, suggesting the presence of tidal airway closure, is abolished after treatment, showing a reopening of small airways during tidal breathing. 


\section{COPD}

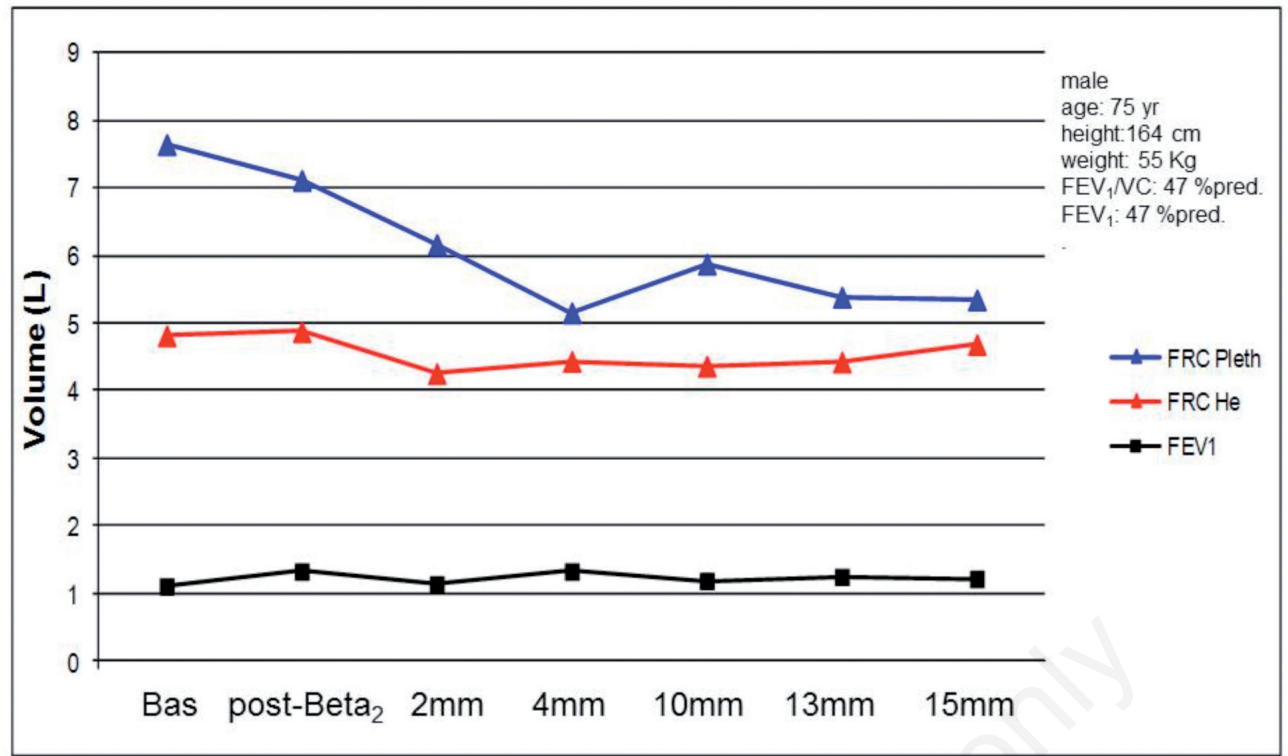

Figure 5. Changes in FRC,Pleth and FRC,He observed in a representative COPD patient with COPD exacerbation, at baseline and during therapeutic follow-up. The marked difference between FRC,Pleth and FRC,He at the beginning, reflecting the presence of tidal airway closure, is reduced but not completely abolished with treatment, suggesting a partial reopening of small airways during tidal breathing. Note the decrease in dynamic pulmonary hyperinflation with treatment as demonstrated by the plethysmographic FRC reduction. Bas = baseline; $\mathrm{mm}=$ months; post-Beta ${ }_{2}=$ after acute administration of $400 \mathrm{mcg}$ of salbutamol by MDI plus spacer.

\section{References}

1. Parè PD and Mitzner W. Airway-parenchymal interdependence. Comprehensive Physiology 2012;2:1921-35.

2. Sanderson RJ, Paul GW, Vatter AE, Filley GF. Morphological and physical basis for lung surfactant action. Respir Physiol 1976; 27:379-92.

3. Milic-Emili J, Torchio R, D’Angelo E. Closing volume: a reappraisal (1967-2007). Eur J Appl Physiol 2007; 99:567-83.

4. Leblanc P, Ruff F, Milic-Emili J. Effects of age and body position on "airway closure" in man. J Applied Physiol 1970;28:448-51.

5. Vlahakis NE, and Hubmayr RD. Response of alveolar cells to mechanical stress. Curr Opin Crit Care 2003;9:2-8.

6. 0'Neill LAJ. TLRs play good cop, bad cop in the lung. Nat Med 2005; 11:1161-2.

7. Vlahakis NE, Schroeder MA, Limper AH, Hubmayr RD. Stretch induces cytokine release by alveolar epithelial cells in vitro. Am J Physiol 1999; 21:L167-73.

8. D’Angelo E, Pecchiari M, Baraggia P, et al. Low-volume ventilation causes peripheral airway injury and increase airway resistance in normal rabbits. J Appl Physiol 2002;92:949-56.

9. Tschumperlin DJ, Shively JD, Kikuchi T, Drazen JM. Mechanical stress triggers selective release of fibrotic mediators from bronchial epithelium. Am J Respir Cell Mol Biol 2003;28:142-9.

10. D'Angelo E, Pecchiari M, Della Valle P, et al. Effects of mechanical ventilation al low lung volume on respiratory mechanics and nitric oxide exhalation in normal rabbits. J Appl Physiol 2005; 99:433-4.

11. Mauad T, Luis LFF, Santos MA, et al. Abnormal alveolar attachments with decreased elastic fiber content in distal lung in fatal asthma.
Response of alveolar cells to mechanical stress. Am J Respir Crit Care Med 2004; 170:857-62.

12. Hamid Q, Song Y, Kotsimbos TC, et al. Inflammation of small airway in asthma. J Allergy Clin Immunol 1997;100:44-51.

13. Pini L, Hamid Q, Shannon J, et al. Difference in proteoglycan deposition in the airways of moderate and severe asthmatics. Eur Resp J 2007;29:71-77.

14. Salerno FG, Pinelli V, Pini L, et al. Effect of PEEP on induced constriction is enhanced in decorin-deficient mice. Am J Physiol Mol Lung Cell Physiol 2007;293:L1111-7.

15. King GG, Eberl S, Salome CM, et al. Differences in airway closure between normal and asthmatic subjects measured with single-photon emission computed tomography and technegas. Am J Respir Crit Care Med 1998;158:1900-6.

16. Samee S, Altes T, Powers P, et al. Imaging the lungs in asthmatic patients by using hyperpolarized helium- 3 magnetic resonance: assessment of response to methacholine and exercise challenge. J Allergy Clin Immunol 2003;111:1205-11.

17. Gibbons WJ, Sharma A, Lougheed D, Macklem PT. Detection of excessive bronchoconstriction in asthma. Am J Respir Crit Care Med 1996;153:582-589.

18. Tantucci C, Bottone D, Borghesi A, et al. Methods for measuring lung volumes: is there a better one? Respiration 2016;4:273-80.

19. Tantucci C, Guerini M, Boni E, et al. Tidal airway closure during broncho-constriction in asthma: usefulness of lung volume measurements. Journal of Asthma 2011;48:33-40.

20. Agusti A and Soriano JB. Dynamic hyperinflation and pulmonary inflammation: a potential relevant relationship ? Eur Resp Rev 2006;5:68-71.

21. Gatta D, Aliprandi G, Pini L, et al. Dynamic pulmonary hyperinflation and low grade systemic inflammation in stable COPD patients. Eur Rev Med Pharmacol Sci 2011;15:1068-73. 